\title{
Predictors and Outcome of Severe Dengue Illness in Children
}

\author{
Sheela Theparambil Alikunjuํㅜ, Nithya Thuruthiyath², Alok Kanjhoor Premdas ${ }^{3}$, Rose Mary Jose ${ }^{4}$ \\ ${ }^{1}$ Department of Paediatrics, Government Medical College, Thrissur, Kerala, India. ${ }^{2}$ Department of Paediatrics, Jubilee \\ Mission Medical College, Thrissur, Kerala, India. ${ }^{3}$ Department of Community Medicine, Government Medical College, \\ Thrissur, Kerala, India. ${ }^{4}$ Department of Paediatrics, Government Medical College, Thrissur, Kerala, India.
}

\section{ABSTRACT}

\section{BACKGROUND}

Dengue fever has become a major public health concern during the last few years with an alarming increase in the incidence in 2017. The state of Kerala was one among the top of the list in India reported to have severe dengue infection. The two main districts in Kerala with high prevalence were Thiruvananthapuram and Palakkad, of which latter is the main referring area to our institution. This study was conducted to evaluate the clinical and demographic profile of children affected with dengue fever and determine the predictors of severity.

\section{METHODS}

This is a hospital based prospective study done at the Department of Paediatrics, Government Medical College, Trissur, during the Monsoon season in 2017. Children diagnosed to have Dengue fever and confirmed by NS1 Ag test or IgM Elisa were included. Children were classified into 3 clinical groups and compared. Comparison was also made in 2 groups as dengue with warning signs and severe dengue as per WHO 2015 clinical guidelines, to find out the predictors of severity. Qualitative data was analysed and expressed in proportions and quantitative data in mean and standard deviation. Chi-square $\left(\chi^{2}\right)$ test was used to evaluate the association between qualitative variables and ANOVA for quantitative variables. $p$ value less than 0.05 was considered significant. SPSS 16.0 software was used for statistical analysis.

\section{RESULTS}

A total of 235 children were included. 77 children (32.9\%) had mild dengue fever,

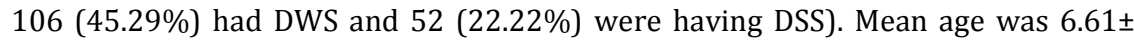
3.497 years. 19 cases (8.2 \%) were Infants below 1 year of age. Majority of children had normal nutritional status. Myalgia, tiredness, vomiting, diarrhoea, abdominal pain, flushing, bleeding, oedema, hypotension, were found to be the common clinical manifestations. Thrombocytopenia, elevated serum hepatic enzymes both SGOT and SGPT, abnormal renal function tests, low sodium, hypoalbuminemia, hypoglycaemia, abnormal radiological findings were found to be the predictors of severity. We had many cases of expanded dengue syndrome including 3 cases of Hemophagocytic lymphohistiocytosis (HLH). The mortality was $0.6 \%$.

\section{CONCLUSIONS}

Dengue fever can affect children irrespective of their age or nutritional status. Older children and male sex were found to be more affected. There are definite clinical and lab parameters which can predict the severity in Dengue fever. Though severe illness is associated with high morbidity early diagnosis and timely appropriate clinical management, correction of dehydration along with proper referral system can save the children. The mortality can be reduced to zero even in patients having expanded dengue syndrome and Dengue shock syndrome. None of the comorbidities had affected the outcome.

\section{KEY WORDS}

DWS (Dengue with Warning Signs), DSS (Dengue Shock Syndrome), AST (Aspartate Aminotransferase) (SGOT), HLH (Hemophagocytic Lymphohistiocytosis)
Corresponding Author: Dr. Sheela Theparambil Alikunju, $S A D^{\prime} R, 55 / 1188$, Aliya Gardens, Panjikal, Ayyanthole, Thrissur-680003, Kerala. India. E-mail: sheelatheparambil@gmail.com

DOI: $10.14260 /$ jemds/2020/143

Financial or Other Competing Interests: None.

How to Cite This Article: Alikunju ST, Thuruthiyath N, Premdas AK, et al. Predictors and outcome of severe dengue illness in children. J. Evolution Med. Dent. Sci. 2020;9(09):655-661, DOI: 10.14260/jemds/2020/143

Submission 18-12-2019,

Peer Review 03-02-2020,

Acceptance 11-02-2020,

Published 02-03-2020.

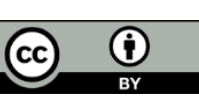




\section{BACKGROUND}

Dengue fever is a serious mosquito-borne viral disease which in recent years has become a major public health concern. The disease is endemic in more than 100 countries and approximately 50-100 million individuals are infected annually and around 24,000 deaths are estimated to occur.(1) Treated DHF/DSS is associated with $1 \%$ mortality rate while untreated cases it comes to $20 \%$, the case fatality rate being $5 \%$.(2) India is one of the seven identified countries in the South-East Asia region regularly reporting outbreaks of Dengue fever with an annual incidence 7.5 to 32.5 million..$^{(+)}$ For the past 10 years the number of cases have gradually increased with an alarming increase in incidence reported during 2016- 2017 and Kerala was on the top of the list. The complex interaction among the host, vector and the virus that are influenced by climatic conditions determine the incidence of the disease.

Dengue viruses (DV) belong to family Flaviviridae and there are four serotypes of the virus referred to as DV-1, DV-2, DV-3 and DV-4. Aedes albopictus was found to be the most abundant vector, followed by Aedes aegypti. DENV-2 is the prevailing serotype.(4) $\mathrm{A}$ global pandemic is represented by all 4 serotypes of the virus. $75 \%$ of the population exposed to the illness are in the Asia Pacific region. (5) Case fatality rate is high in patients with severe dengue infection which consists of dengue haemorrhagic fever (DHF) and dengue shock syndrome (DSS). Early preventive measures and improved clinical management has substantially lowered the mortality from $20 \%$ to less than $1 \%$ during the past decade.(6)

Dengue affects all age groups and infection with any one serotype confers lifelong immunity to that serotype.(5) The re infection is associated with elevated risk and observed to be more severe in children due to immunological phenomenon.(6) In India, dengue is endemic in 29 states according to the annual report of the Department of Health and Family Welfare 2017-18(7) The highest number were reported from West Bengal followed by Tamil Nadu and Kerala. Approximately 18,700 cases of Dengue have been confirmed from India. ${ }^{\left({ }^{8}\right)}$ The total number of patients in Kerala was 9104 followed by Tamil Nadu \& Karnataka as states with highest incidence. ${ }^{(9)}$ The early onset of Monsoon during the year was accompanied by a rush of many communicable diseases. In the list of Dengue hot spot districts published by the health department, Trivandrum tops the chart followed by Kollam, Palakkad, Malappuram, Kozhikode and Kannur.(10)

We wanted to study the clinical and epidemiological profile of dengue fever in children. We also wanted to study the predictors and outcome of severe dengue illness by comparing the clinical and lab parameters in children having dengue with warning signs and severe dengue.

\section{METHODS}

This is a prospective study conducted in the Department of Paediatrics, Government Medical College, Thrissur, a tertiary care centre in central Kerala from March 2017 to September 2017 (monsoon season in Kerala).

\section{Sample Size Calculation}

Sample size was calculated using the formula $\mathrm{N}=(\mathrm{Z} \alpha+\mathrm{Z} \beta)^{2} 2 \mathrm{SD}^{2} / \mathrm{d}^{2}$

where, $\mathrm{Z} \alpha=2.576$, a constant for $\alpha=1 \% \mathrm{Z} \beta=1.64$, a constant for $\beta=5 \%$, Power of the study=1- $\beta=95 \%$,

$\mathrm{SD}=\left(\mathrm{SD}_{1}+\mathrm{SD}_{2}\right) / 2 \mathrm{~d}=\mu_{1}-\mu_{2}=$ difference between means

As per the reference study(16) Clinical profile and outcome of children admitted with dengue fever in a tertiary care hospital in South India'. by Kumar BV, Simna L, Kalpana D, Kailas L. (From departments of Paediatrics and Paediatric Neurology, GMC Trivandrum, Kerala, India)

$\mathrm{SD}_{1}=115 \mathrm{SD}_{2}=27 \mu_{1}=920 \mu_{2}=98$ (from SGPT values of the reference study)

$\mathrm{N}=(\mathrm{Z} \alpha+\mathrm{Z} \beta)^{2} 2 \mathrm{SD}^{2} / \mathrm{d}^{2}=218$

\section{Inclusion Criteria}

Children admitted to Paediatric ward or ICU diagnosed as Dengue fever with clinical features and laboratory parameters were selected. Those confirmed on consecutive sampling by NS1Ag test or IgM Elisa were included.

\section{Exclusion Criteria}

Those children with other chronic illnesses diagnosed to have Dengue fever.

Results of laboratory parameters like complete blood count, including packed cell volume, platelet count, LFT,RFT,RBS, Serum calcium and Electrolytes, were collected. Coagulation profile, serum IgG, ultrasound abdomen, MRI, and chest radiography were performed only if clinically indicated. Children were classified according to WHO guidelines 2015.

Atypical features and complications with immediate outcome measures were noted.(5) Patients were followed up daily to assess clinical and laboratory progression till discharge and subsequently in outpatient care if required. Children with chronic co morbidities were also observed and analysed separately to know the outcome.

\section{Statistical Analysis}

The statistical analysis was done using SPSS. Qualitative data was analysed and expressed in proportions and quantitative data in mean and standard deviation. The discrete variables were analysed using Chi-Square test, and continuous variables using ANOVA. The analysis was performed using SPSS 16.0 software. $p$ value less than 0.05 was considered as significant.

\section{RESULTS}

A total of 235 children were included of which 132(56.2\%) were boys and 103 (43.8\%) were females. (M: $F=1.28: 1)$.The highest number of admissions were from the district of Palakkad, 164 - (69.7\%) followed by Thrissur 63-(26.8\%) and Malappuram 8-(3.5\%). Admissions were maximum in the month of June (68) followed by July (57) and August (53). 0-1 age group included 19 infants. (8.1\%) and 39 (16.5\%) were more than 10 years of age. Mean age of the series was 6.61 (SD 3.49) years and 19 (8.2\%) were infants. Mild Protein energy malnutrition in 41 children, grade 2 PEM in 5 cases and grade 4 PEM in two children. 


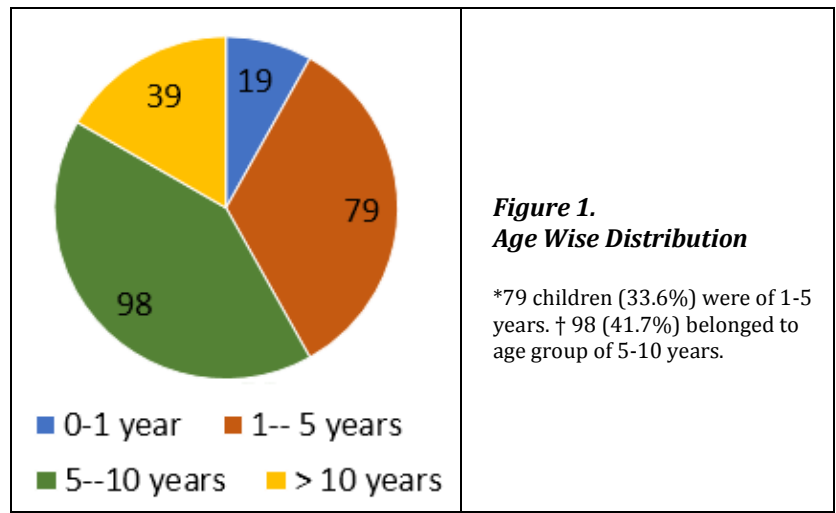

Clinical classification among the study group showed 77 (32.9\%) had mild dengue fever, 106 (45.29\%) had dengue with warning signs and $52(22.22 \%)$ had severe dengue fever. Dengue shock syndrome was present in 17 children. Children were initially compared in 3 groups as mild dengue, Dengue with warning signs, and severe dengue. Comparison was also made in 2 groups as mild $167-(71.4 \%)$ and severe dengue (67$28.6 \%$ ) as per WHO 2015 clinical guidelines to know the predictors of progression to severe dengue. Multiple variables were also compared in dengue with warning signs and severe dengue.

Clinical manifestations included Rash $(40.7 \%)$ myalgia (53.3\%), arthralgia (26.4\%), vomiting (62.2\%), diarrhoea $(12.5 \%)$, abdominal pain $(63.8 \%)$, headache $(37.7 \%)$ generalised lymphadenopathy (23.8\%), oedema (28.8\%), lethargy $(48.1 \%)$ flushing $(47.5 \%)$ bleeding manifestations (32.2\%). Hess test was positive in $25.21 \%$ Mucosal bleeds were manifested in $(25.7 \%)$ children. Bradycardia (10.5\%) Hypotension $(21.9 \%)$ \& manifestations in the eyes $(39.8 \%)$. Laboratory investigations revealed anaemia (17.9\%), thrombocytopenia (83.8\%), Leucopenia (59.9\%), Relative lymphocytosis $(67.6 \%)$ leucocytosis $(6.1 \%)$, elevated hepatic enzymes (55.7\%), Abnormal RFT (4.1\%), hyponatremia (26.5\%) hypoalbuminemia (24.7\%) Dengue NS1 antigen was positive in (91.6\%) and IgM dengue positivity was shown in (85.7\%)

Fever was present in almost all children with an average duration of 4.8 days. $p$ value was found to be significant for vomiting, diarrhoea, abdominal pain, bleeding manifestations including mucosal bleed, positive Hess test, excessive tiredness, hypotension flushing and eye changes when mild and severe dengue were compared. Among the lab parameters. $p$ value were found to be significant for platelet count, liver function tests, renal function tests, serum sodium, serum albumin and radiological changes. Leucopenia, relative lymphocytosis and elevated PCV were not found to be significant.

The changes in the eye observed were periorbital puffiness, sub conjunctival haemorrhage, Retro orbital pain, and macular haemorrhage. Independent predictors were found to be abdominal pain or tenderness, persistent vomiting, lethargy, mucosal bleed, clinical fluid accumulation, hepatomegaly $>2 \mathrm{~cm}$, platelet count $<100 \times 10^{9} / \mathrm{L}$. Outcome measure of severe dengue were defined as per WHO guidelines 2015. Mean duration of hospital stay $4.17+(2.613)$ days.

Radiological evaluation of these children revealed Pleural effusion in 33 cases (16 had bilateral \&unilateral effusion in 17 cases with predominant right sided involvement. 28 children had ascites. Perinephric collection and severe hemoperitoneum were seen in 3 children. 2 children had pericardial effusion. The radiological findings seen in gall bladder included cholecystitis, ${ }^{(8)}$ Cholangitis ${ }^{(9)}$ Peri gall bladder collection(4) Gall bladder wall oedema and sludge in bladder.(3) PT and APTT values were deranged in 42 cases among the severely affected group. Isolated PT prolongation was seen in 9 cases (17.3\%), APTT prolongation in 19 (38\%) while both were deranged in 12 (23\%) of dengue shock syndrome. AST (SGOT) values $>100$ units in 36 cases of severe dengue with values observed more than 3000 in 3 cases.

\begin{tabular}{|c|c|c|c|c|}
\hline Parameter & Variable & DWS & DSS & p Value \\
\hline Platelet count & $\begin{array}{l}<50000 / \text { cu.mm } \\
50000-1 \text { lakh } \\
1-1.5 \text { lakhs } \\
>1.5 \text { lakh }\end{array}$ & $\begin{array}{l}23 \\
53 \\
19 \\
11\end{array}$ & $\begin{array}{l}27 \\
19 \\
5 \\
1\end{array}$ & 0.001 \\
\hline Serum Sodium & $\begin{array}{l}<130 \mathrm{meq} / \mathrm{L} \\
\quad \begin{array}{l}130-135 \\
>135\end{array}\end{array}$ & $\begin{array}{l}15 \\
19 \\
21\end{array}$ & $\begin{array}{l}20 \\
11 \\
10\end{array}$ & 0.000 \\
\hline SGOT & $\begin{array}{c}<200 \text { units } \\
>200\end{array}$ & $\begin{array}{c}76 \\
6\end{array}$ & $\begin{array}{l}35 \\
12\end{array}$ & 0.005 \\
\hline Serum albumin & $\begin{array}{c}<2.5 \mathrm{gm} / \mathrm{dl} \\
2.5-3\end{array}$ & $\begin{array}{l}16 \\
59\end{array}$ & $\begin{array}{l}29 \\
19\end{array}$ & 0.000 \\
\hline RBS & $\begin{array}{c}40-70 \mathrm{mg} / \mathrm{dl} \\
>70 \mathrm{mg} / \mathrm{dl}\end{array}$ & $\begin{array}{l}64 \\
41\end{array}$ & $\begin{array}{c}104 \\
25\end{array}$ & 0.001 \\
\hline \multicolumn{5}{|c|}{$\begin{array}{c}\text { Table 1. Multiple Comparison of Lab Parameters in } \\
\text { DWS and Severe Dengue }\end{array}$} \\
\hline \multicolumn{5}{|c|}{$\begin{array}{l}\text { *Multiple comparison in type } 2 \text { dengue with warning signs and type } 3 \text { severe } \\
\text { dengue fever. } † \text { Significant association with, platelet count, RBS, Serum sodium, } \\
\text { RBS, Serum albumin, and SGOT. } \neq \text { The mean values are found to be high in Severe } \\
\text { dengue. }\end{array}$} \\
\hline
\end{tabular}

\begin{tabular}{|c|c|c|c|}
\hline Lab Parameter & Mild & DWS & Severe Dengue \\
\hline $\mathrm{Hb}$ & $12.13 \pm 1.186$ & $11.99 \pm 1.80$ & $11.97 \pm 1.96$ \\
\hline $\mathrm{PCV}$ & $34.67 \pm 7.72$ & $36.57 \pm 4.13$ & $35.96 \pm 7.49$ \\
\hline Platelet count & $52430 \pm 6055$ & $90613.21 \pm 50028.67$ & $58653.85 \pm 36686.2$ \\
\hline SGOT & $153.55 \pm 151.93$ & $213 \pm 429.57$ & $469.39 \pm 794.15$ \\
\hline SGPT & $77.84 \pm 69.49$ & $99.32 \pm 162.87$ & $165.49 \pm 281.28$ \\
\hline Serum sodium & $134 \pm 3.4$ & $133.95 \pm 8.157$ & $129.88 \pm 5.853$ \\
\hline Serum albumin & $2.283 \pm 0.147$ & $3.055 \pm 0.458$ & $2.194 \pm 0.55$ \\
\hline \multicolumn{4}{|c|}{ Table 2. The Mean Values of Lab Parameters in Mild, DWS } \\
(Dengue with Warning Signs) \& Severe Dengue \\
\hline \multicolumn{4}{|c}{} \\
\hline
\end{tabular}

The lab parameters which were associated with severity of dengue fever were compared in groups using ANOVA. Significant association was present for values of platelets, SGOT, Serum sodium Serum albumin.

\begin{tabular}{|c|c|c|}
\hline Parameter & F Value & Significance \\
\hline $\mathrm{Hb}$ & .540 & .583 \\
\hline $\mathrm{PCV}$ & 1.826 & .164 \\
\hline Platelet & 21.56 & .000 \\
\hline SGOT & 6.249 & .002 \\
\hline SGPT & 3.125 & .049 \\
\hline Serum Sodium & 7.029 & .001 \\
\hline Serum Albumin & 23.167 & .000 \\
\hline \multicolumn{2}{|c|}{ Table 3. Comparison of The 3 Clinical Groups Using ANOVA } \\
\hline $\begin{array}{l}\text { *Significant p value for Platelet count, Serum sodium Serum Albumin and SGOT } \\
\text { values when compared in 3 groups }\end{array}$
\end{tabular}




\begin{tabular}{|c|c|c|}
\hline Dependent Variable & Type & Significance \\
\hline Platelet count & $\begin{array}{l}12 \\
13 \\
23\end{array}$ & $\begin{array}{l}.001 \\
.000 \\
.000\end{array}$ \\
\hline Serum sodium & $\begin{array}{l}12 \\
13 \\
23\end{array}$ & $\begin{array}{l}.170 \\
.001 \\
.068\end{array}$ \\
\hline Serum albumin & $\begin{array}{l}12 \\
13 \\
23\end{array}$ & $\begin{array}{l}.094 \\
.000 \\
.000\end{array}$ \\
\hline SGOT & $\begin{array}{l}12 \\
13 \\
23\end{array}$ & $\begin{array}{l}1.00 \\
.003 \\
.010\end{array}$ \\
\hline
\end{tabular}

Post hoc analysis showed Serum sodium values are significantly associated with mild \&severe dengue. Serum albumin values are also significant when mild and severe dengue were compared. $p$ value was not significant when comparing dengue with warning signs and mild dengue. Platelet count shows significant association from mild to dengue with warning signs and severe dengue. SGOT values shows significant association with severe dengue.

\begin{tabular}{|c|c|c|}
\hline System Involved & Unusual Atypical Manifestations & Number \\
\hline \multirow[t]{5}{*}{ Neuromuscular } & Encephalopathy & 4 \\
\hline & Transient weakness & 2 \\
\hline & Myositis with high $\mathrm{CPK}$ & 2 \\
\hline & Febrile seizures & 6 \\
\hline & ADEM & 1 \\
\hline \multirow[t]{4}{*}{ Cardiovascular } & Arrhythmias- transient 2:1 block & 1 \\
\hline & Bradycardia with ectopics & 2 \\
\hline & Myocarditis & 2 \\
\hline & Pericardial Effusion & 1 \\
\hline \multirow[t]{4}{*}{ Respiratory } & Severe LRTI - ventilator support & 9 \\
\hline & ARDS & 4 \\
\hline & Pleural effusion & 33 \\
\hline & Collapse lung & 3 \\
\hline \multirow[t]{4}{*}{ Gastrointestinal } & Hepatic involvement & 41 \\
\hline & Fulminant hepatic failure & 4 \\
\hline & cholecystitis & 8 \\
\hline & Clinical jaundice & 3 \\
\hline \multirow[t]{2}{*}{ Haematological } & Coagulopathy & 42 \\
\hline & HLH & 3 \\
\hline \multirow[t]{2}{*}{ Eyes } & Subconjunctival bleed & 4 \\
\hline & Macular haemorrhage with partial visual loss & 1 \\
\hline \multirow[t]{3}{*}{ Renal } & AKI & 4 \\
\hline & HUS & 2 \\
\hline & MODS & 4 \\
\hline \multicolumn{3}{|c|}{ Table 5. Expanded Dengue Syndrome } \\
\hline
\end{tabular}

\section{DISCUSSION}

Compared to previous years, there was a significant rise in number of admissions during 2017 followed by a decline in the incidence thereafter. Maximum admissions were during the month of July and August. The monsoon showers had hit early in Kerala since April and there was a rush of many communicable diseases (10).Most of the cases were referred from Palakad district.(11)In the present study it was found that males were more affected with a male to female ratio of 1.28:1 as reported in most of the studies(12) A few studies have reported more incidence in females $(12,13)$

Mean age of the group was $6.61 \pm 3.497$ years. Infants constituted $8.2 \%$ and $57.7 \%$ of children belonged to $5-15$ age group in our study group similar to that seen in study conducted by Manjunadh et al. ${ }^{12)}$ Study by Mittal et al showed $68 \%$ cases were in the age group of $6-12$ years, only $2.9 \%$ were infants and the mean age group was $8.3 \pm 3.5$ years (14) A study conducted by Misra S et al in 2016 also shows similar results.(15) Among the group 4 infants belonged toless than 6 months of age but the age group showed no association with severity of illness when the different age groups were compared.

Only $22.4 \%$ children had protein energy malnutrition and most of the children had normal weight. It has been reported that children having better nutritional status can also have more complications due to better immunological response.(16)

Among the study group, 77 had mild dengue fever, 106 had dengue with warning signs and 52 had severe dengue fever as per the latest WHO classification (5)There was an overlapping of clinical signs among Dengue with warning signs, severe Dengue, including expanded dengue syndrome and severe dengue shock syndrome(DSS)as described in WHO national guidelines.

Fever was present in almost all the cases (94.8\%) and the mean duration was 4.8 days similar as described in most of the studies. (11).vomiting and abdominal pain, myalgia, lethargy, rash, Flushing, bleeding manifestations were the most common symptoms at presentation. Most of the studies have shown similar data of clinical manifestations as described in a study conducted in Karachi, Pakistan.(17)and from Kerala(11,18)

Generalized lymph node enlargement was seen in (23.8\%) with features of lymph node abscess in 3 children. A study from Trivandrum(16) shows less incidence of lymphadenopathy. Hepatomegaly alone was seen in 73 children, 46 had hepatosplenomegaly with enlarged tender liver in 14 cases and 8 had splenomegaly alone. These findings were significantly associated with severe dengue with a $\mathrm{p}$ value of 0.002 , similar to the results described by Mohan et al.(13,19) Clinical jaundice was present only in 3 children similar to that reported in the study from Trivandrum.(16) Severe dehydration was present in 12 children and among them 9 had diarrhea. The presentation was with severe pneumonia in 8 children and 4 had features of ARDS.

Leucopenia was seen in $59.9 \%$ and Thrombocytopenia in 83.8.\% which was similar to other prospective observational studies.(18) The mean value of PCV with mild dengue was 35.8 and 36.36 in mild and severe dengue respectively. In our series the elevated values are seen in both severe dengue and dengue with warning signs without any signs of plasma leakage similar to a study by Mohan et al.(19) Many studies have shown significant association of elevated haematocrit. Our study series showed elevated mean hematocrit in both DWS and DSS but the $\mathrm{p}$ value was not found to be significant. 5 children had fall in PCV requiring blood transfusion. 
Independent predictors were found to be abdominal pain or tenderness, persistent vomiting, excessive tiredness, mucosal bleed, clinical fluid accumulation and hepatomegaly. The presence of hepatosplenomegaly was found to be significant when dengue with warning signs and severe dengue fever were compared (p.0.018). The independent lab predictors while comparing mild and severe dengue included, low platelet count, leucopenia, elevated hepatic enzymes as evident from their mean values and significant $\mathrm{p}$ values. Similar results are shown in other studies.(16) Significant difference in AST among dengue fever without warning signs, DWS and SD $(\mathrm{p}<0.001)$ similar to other studies (20) DWS and severe dengue when compared showed platelet count, Serum Sodium, Albumin, Elevated, SGOT, Serum glucose, Renal function test, and coagulation profile as predictors.(20) We could not compare the coagulation profile in DWS and severe dengue since the test was not routinely done.(21) The mean values of PT and a PTT (19 and 46) respectively were found to be very high in severe dengue. Hypocalcaemia was present in 6 children, among them 2 had clinical manifestations.

Children with severe disease had $51.5 \%$ chest $\mathrm{X}$ ray findings compared to $24.3 \%$ of mild disease and USG findings were present in $59.9 \%$ of severe disease with a $p$ value of 0.002. LFT were found significantly high in severe dengue cases, (20) $85.7 \%$ of children showed IgM positivity among 133 cases. NS1 was positive in 196/214 cases(91\%)as we had included patients with NS1 or Ig M Positive cases in the study.(22) Ig G was sent only for 4 cases of severe dengue out of which 3 were found to be positive. Combination of the NS1 antigen and antibody tests could increase the diagnostic efficiency for early diagnosis of dengue infection(23) Radiological signs were more seen in severe dengue cases. The changes seen in the gall bladder was similar to that described in the study conducted by Setiavan et al.(24)

Many children had features of expanded dengue syndrome as described in the 2015 guidelines. Neurological manifestations included altered sensorium with features of encephalopathy, febrile seizures, ADEM, Facial palsy and severe proximal myositis with highly elevated CPK similar to a study conducted by Kammath et al.(25) 3 children had features of Hemophagocytic lymphohistiocytosis. The age of the affected children were in the range of 6 months to 5 years. All were apparently normal and had serological evidence (NS1 Antigen or Dengue IgM antibodies). Evidence of plasma leakage in one child and Dengue Shock Syndrome (DSS) in 2 children. All the 3 children fever more than 7 days with elevated hepatic enzymes showing hepatosplenomegaly, edema and ascites. A 6 month old infant was the worst affected with features of MODS, Altered sensorium, ARDS, Coagulopathy, ARF, and DIC. Investigations revealed Serum Ferritin ( $>20000 \mathrm{ug} / \mathrm{L}$ ) Hypertriglyceridemia $(1750 \mathrm{mg} / \mathrm{dl})$ Fibrinogen $(<50 \mathrm{mg} / \mathrm{dL})$ and elevated LDH. Baby was treated with Methyl prednisolone IV Ig, cyclosporins, prolonged ventilator support along with other supportive measures for 3 weeks. She had an uneventful recovery. Other 2 children with HLH were also had responded well to steroids alone, similar reports in a study from Srilanka.(26) Cardiac manifestations included myocarditis, (2) arrythmia with 2:1 block in 2 children, pericardial effusion in one child. A study conducted by
Sheethal(27) has shown similar results and report myocardial involvement is one among the predictors of severity.

83 children required management in ICU (39.1 $\%)$.Intravenous fluids were required for 110 children as per the latest guidelines. Hourly ORS and or salted Rice water was recommended for all cases of mild disease. Other supportive measures like Antibiotics for co existent infections,(17) Platelet concentrates(14) $^{(14)}$ packed cells(8) FFP(14) Methyl prednisolone(4) Hydroxyethyl $\operatorname{starch}^{(2)}$ inotropes(9) were used as recommended. An 8 yr old girl child who had all confirmatory clinical and lab features of dengue IgM and NS1 showed persistently elevated ESR and hip arthritis. ECHO showed evidence of acute rheumatic fever with acute severe MR. We had many children with comorbidities who were excluded from the study group and analysed separately. Haemophilia with inhibitor on FEIBA, Thalassemia Major, Nephrotic syndrome(FSGS), seizure disorder, Microcephaly with seizures, Congenital heart disease with VSD \& ASD were the comorbidities. All the children in whom 2 had severe illness also responded well.

Mean duration of hospital stay was $4.17+2.613$ days and this was seen significantly associated with severity of illness. ( $p$ value 0.000 ) when mild and severe dengue were compared. Coagulopathy and acute kidney injury in patients with dengue viral illness is associated with increased hospital stay.(28) The average platelet recovery time among 206 cases was 2.9 (SD 1.131) days similar to a study from Trivandrum.

All cases of severe dengue had recovered except a 9 month old infant who had been admitted with MODS, ARDS and Coagulation failure. Acute kidney injury in DHF and DSS are associated with high mortality as described in a study conducted by Khalil et al. High mortality associated with severe dengue is also been reported in a few studies.(29) Though we had many cases with refractory complications referred to our tertiary care institution the mortality rate was only $0.6 \%$.A study conducted by Banerjee et al also report the mortality is only $1.5 \%$.(30) $^{30}$

\section{CONCLUSIONS}

Dengue fever can affect children irrespective of their age or nutritional status. Older children and male sex were found to be more affected. There are definite clinical and lab parameters which are predictors of the severity of Dengue fever. Though severe illness is associated with high morbidity early diagnosis and timely appropriate clinical management, correction of dehydration along with proper referral system can save the children. The mortality can be reduced to zero even in patients having expanded dengue syndrome and Dengue shock syndrome. None of the comorbidities had affected the outcome.

\section{ACKNOWLEDGEMENT}

We thank the parents of study participants for their cooperation during the study. We would like to acknowledge 
all the staff members from Departments of Paediatrics, Microbiology and Social \& Preventive Medicine for their help and support.

\section{REFERENCES}

[1] World Health Organization. Dengue and dengue haemorrhagic fever. Fact Sheet. No. 117, 2002. http//www.who.int/mediacentre/factsheets/fs117/en/

[2] World Health Organization. Global Strategy for Dengue Prevention and Control: WHO 2012-2020. Geneva: World Health Organization, 2012.

[3] Gupta N, Srivastava S, Jain A, et al. Dengue in India. Indian Journal of Medical Research 2012;136(3):373-90.

[4] National Vector Borne Disease Control \& Prevention. Dengue. Dengue Cases and Death in the Country since 2010. www.nvdcp.gov.in/den-cd.html Accessed on 30 April 2017.

[5] National Guidelines for Clinical Management of Dengue Fever.

2015. http://www.searo.who.int/india/publications/national_ guidelines_clinical_management_dengue1.pdf

[6] Wichmann O, Hongsiriwon S, Bowonwatanuwong C, et al. Risk factors and clinical features associated with severe dengue infection in adults and children during the 2001 epidemic in Chonburi, Thailand. Tropical Medicine \& International Health 2004;9(9):1022-9.

[7] Das B, Das M, Dwibedi B, et al. Molecular investigations of dengue virus during outbreaks in Orissa state, Eastern India from 2010 to 2011. Infection, Genetics and Evolution 2013;16:401-10.

[8] Ministry of Health and Family Welfare, Govt. of India, Annual report of department of health and family Welfare 2017-18. Tech. Rep, New Delhi, India, 2019. https://mohfw.gov.in/basicpage/annual-reportdepartment-health-and-family-welfare-2017-18.

[9] Dengue cases in India: Kerala leads the list. Kerala News. New Delhi. 2017. http://www.deepikaglobal.com/Kerala.aspx? Denguecases-in-India:-Kerala-leads-the-list.46225 Accessed on 4th April 2018.

[10] Dengue cases swell in Kerala. Thiruvananthapuram. The Hindu.

2017.

http://www.thehindu.com/news/cities/Thiruvananthap uram/dengue-fever-cases-swell-

inkerala/article19089841.ece\#! Accessed at 17 July 2017.

[11] Kumar SK, Rajendran NK, Brabhukumar AC. Clinical profile of dengue fever in children: analysis of 2017 outbreak from Central Kerala, India. Int J Contemp Pediatr 2018;5(6):2265-9.

[12] Manjunath MN, Chaithanya CN, Sharanya R. A study on clinical features and cost incurred by dengue syndrome patients admitted to tertiary care hospital. Br J Med Pract 2015;8(2):a811.
[13] Ratageri VH, Shepur TA, Wari PK, et al. Clinical profile and outcome of dengue fever cases. Indian J Pediatr 2005;72(8):705-6.

[14] Mittal H, Faridi MMA, Arora SK, et al. Clinicohematological profile and platelet trends in children with dengue during 2010 epidemic in North India. Indian J Pediatr 2012;79(4):467-71.

[15] Mishra S, Ramanathan R, Agarwalla SK. Clinical profile of dengue fever in children: a study from Southern Odisha, India. Scientifica (Cairo) 2016;2016:6391594.

[16] Kumar BV, Simna L, Kalpana D, et al. Clinical profile and outcome of children admitted with dengue fever in a tertiary care hospital in South India. Indian J Child Health 2018;5(1):32-7.

[17] Ahmed S, Arif F, Yahya Y, et al. Dengue fever outbreak in Karachi 2006- a study of profile and outcome of children under 15 years of age. J Pak Med Assoc 2008;58(1):4-8.

[18] Kalayanarooj S, Nimmannitya S. Is dengue severity related to nutritional status? Southeast Asian J Trop Med Public Health 2005;36(2):378-84.

[19] Mohan B, Patwari AK, Anand VK. Hepatic dysfunction in childhood dengue infection. J Trop Pediatr 2000;46(1):40-3.

[20] Sahana KS, Sujatha R. Clinical profile of dengue among children according to revised WHO classification: analysis of a 2012 outbreak from Southern India. Indian J Pediatr 2015;82(2):109-13.

[21] Vazhayil PP, Stephen ST, Kumar V. A retrospective observational study of dengue fever in a tertiary care Center in Kerala. Int J Sci Stud 2017;5(1):30-4.

[22] Shrivastava A, Dash PK, Tripathi NK, et al. Evaluation of a commercial Dengue NS1 enzyme-linked immunosorbent assay for early diagnosis of dengue infection. Indian J Med Microbiol 2011;29(1):51-5.

[23] Kassim FM, Izati MN, TgRogayah TA, et al. Use of dengue NS1 antigen for early diagnosis of dengue virus infection. Southeast Asian J Trop Med Public Health 2011;42(3):562-9.

[24] Setiawan MW, Samsi TK, Pool TN, et al. Gallbladder wall thickening in dengue hemorrhagic fever: an ultrasonographic study. J Clin Ultrasound 1995;23(6):357-62.

[25] Kamath SR, Ranjit S. Clinical features, complications and atypical manifestations of children with severe forms of dengue haemorrhagic fever in South India. Indian J Pediatr 2006;73(10):889-95.

[26] Kankananarachchi I, Pradeep N, Munasinghe TM, et al. Haemophagocytic Lymphohistiocytosis (HLH) Secondary to Dengue Haemorrhagic Fever (DHF): A Paeditric Case Series. EC Paediatrics 2018;7(7):638-40.

[27] Sheetal S, Jacob E. A study on the cardiac manifestations of dengue. J Assoc Physicians India 2016;64(5):30-4.

[28] Khalil MAM, Tan J, Khalil MAU, et al. Predictors of hospital stayand mortality in dengue virus infection-experience from agha khan university hospital Pakistan. BMC Res Notes 2014;7:473. 
[29] Van Gorp ECM, Setiati TE, Mairuhu ATA, et al. Impaired fibrinolysis in the pathogenesis of dengue hemorrhagic fever. J Med Virol 2002;67(4):549-54.
[30] Banerjee A, Barik KL, Bandyopadhyay A, et al. A study on the clinical features of dengue virus infected pediatric patients. Int J Contemp Pediatr 2018;5(2):368-71. 